\title{
CONSIDERACIONES METODOLÓGICAS EN TORNO A LA HISTORIA DE LA FILOSOFÍA EN EL RENACIMIENTO
}

Laura Benítez Grobet

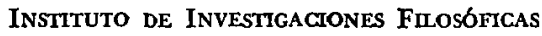
Universidad Nacional autónoma de México

Por eso abundan las historias de la filosofía compuestas de numerosos volúmenes y hasta si se quiere, llenos de erudición y en los que, sin embargo, brilla por su ausencia el conocimiento de la materia misma sobre la que versan. Los autores de tales historias podrian compararse a animales, por cuyos oidos entran todos los sonidos de la música, pero sin ser capaces naturalmente de captar una cosa, la armonía de los sonidos. ${ }^{1}$

Cualquier intento por acercarse a la historia de la filosofía remite a la necesidad de explicitar los conceptos mismos de historia y de filosofía, cuya vinculación ha parecido a muchos autores difícil de establecer. ${ }^{2}$ Así, en el curso de esta exposición me propongo aclarar cómo entiendo la conexión entre historia y filosofía lo cual me permitirá caracterizar al Renacimiento como periodo filosófico.

Para iniciar el análisis parto del supuesto de la existencia de la actividad filosófica que como toda actividad humana, y en este caso, específicamente cultural, es susceptible de ser historiada. En otros términos, parto del hecho de la historia de la filosofía como una parcela de la historia general humana. No obstante, en la propia constatación del fenómeno surge la necesidad de señalar cuáles son los hechos filosóficos a que tal historia habrá de abocarse, y si es posible determinar alguna 0 algunas regularidades en su marcha a fin de contribuir a la explicación teórica de la historia de la filosofía.

1 Hegel, G. W. F., Lecciones sobre la historia de la filosofia. Traducción directa Wenceslao Roces, F.C. E., México-Buenos Aires, 1955, pp. 6-7.

2 Hegel, L. W. F. en op. cit., p. 14, plantea la dificultad aparente que encierra el rubro Historia de la filosofía por la contradicción que supone el asociar la idea de historia como algo cambiante y contingente a la idea de la filosofía como algo perenne y absoluto. 
Para facilitar el acceso al problema me he propuesto confrontar dos teorías interpretativas de la historia de la filosofía que ofrecen dos alternativas diferentes pero que pueden ser manejadas, en mi concepto, como complementarias.

1. El doble determinismo sistemático en relación con la historia de la filosofia

Llamo doble determinismo sistemático con respecto a la historia de la filosofía, a la concepción hegeliana de que la continuidad histórica de la filosofía es el fruto de la continuidad de los sistemas, que en última instancia adquieren su sentido o vienen a parar en el sistema Hegel, y que sólo los sistemas son estrictamente filosóficos y por ende sólo ellos conforman el caudal histórico de la filosofía.

Habrá que recordar que para Hegel cada momento de la historia de la filosofía resulta necesariamente generado por el anterior y es, a su vez, generador del sucesivo en el proceso dialéctico. "Las oposiciones entre sistemas sucesivos o negaciones que cada uno representa frente al anterior no es una destrucción o aniquilamiento del antecedente sino su superación, la negación conserva lo que niega, le otorga una verdad superior integrándolo con su opuesto en una síntesis más elevada." ${ }^{3}$ Así pues, Hegel da cuenta del desarrollo histórico de la filosofía sobre la base de la concepción de ésta como el momento más elevado en el movimiento de autoconciencia del Espíritu Absoluto y, por otra parte, hace coincidir el desarrollo histórico con el desarrollo dialéctico expuesto en la Lógica de modo que parte "de la identificación de las dos series (lógica-cronológica) afirmándose que a la serie de las ideas que se suceden en la formación dialéctica del sistema hegeliano, debe corresponder la sucesión de los principios inspiradores de los sistemas que se van presentando en la historia". ${ }^{4}$

Hegel revalora la historia de la filosofía que no será más ni el mero "cementerio de los sistemas", ni un puro repertorio erudito de doctrinas; se busca pues la armonia de los componentes propios de la filosofía la cual puede ser bien entendida como su hilo conductor o el sentido propio de esa historia que en Hegel sólo puede verse como sistematicidad de sistemas.

En rigor el cuadro que ofrecen las Lecciones sobre la historia de la filosofia de Hegel es el de la organización sistemática de los sistemas; de ahí que, en cuanto al determinismo sistemático o comprensión de la

3 Mondolfo, Rodolfo, Problemas y métodos de investigación en la historia de la filosofia. Eudeba, Buenos Aires, 1960, p. 42.

4 Mondolfo, Rodolfo, op. cit., pp. 45-46. 
historia de la filosofía como la sucesión integrada de los sistemas, y mejor aún, como la trabazón necesaria de sistemas en el conjunto del desarrollo dialéctico, es claro que:

1. Los sistemas se determinan uno a otro en su correlación sucesiva $\mathrm{y}$ dinámica.

2. Sólo en la totalidad sistemática es posible aprehender el sentido de cada parte que es a su vez un sistema.

3. Por lo cual queda claro que lo que se explica es la trabazón interna de los sistemas puesto que el carácter totalizante de la filosofía hegeliana hace innecesario cualquier otro tipo de explicación.

Por otra parte, en cuanto al determinismo sistemático entendido como que la filosofía sólo puede estar integrada por elaboraciones teóricas traducibles en sistemas y que sólo ellos necesariamente conforman su caudal histórico, es solamente una consecuencia del racionalismo absoluto que exige aplicar sin restricción el postulado de la sistematicidad. Se trata pues del problema de la identificación entre sistema y filosofía o, para ser más claros, de la confusión entre la actividad filosófica que exige por definición la metodicidad y el rigor (sistematicidad) y la obligatoriedad de producir sistemas.

Si toda filosofía es sistema o, lo que es lo mismo, si cualquier elaboración en este campo que quiera ser llamada filosófica debe ser un sistema, entonces tropezamos de inmediato con grandes sectores de la producción filosófica que o bien no son filosóficos o bien, siendo filosóficos, no son sistemas aun cuando estén elaborados rigurosamente, esto es, metódica y sistemáticamente.

El problema del doble determinismo sistemático, a saber, toda producción filosófica debe ser un sistema, por ende la historia de la filosofía es la historia de la concatenación sistemática de los sistemas, es que finalmente deja fuera de tan coherente (racionalista y determinista) explicación grandes parcelas de la actividad filosófica.

Frente a este enfoque existen otras formas de encarar el problema de la continuidad de la filosofía, pero me ha parecido muy a propósito traer a colación la perspectiva problemática que Rodolfo Mondolfo tiene de la historia de la filosofía porque enriquece la explicación teórica a través de sus numerosos e importantes escritos sobre la materia en la actualidad.

\section{El enfoque problemático en relación con la historia de la filosofía}

Al enfocar problemáticamente la historia de la filosofía, su continuidad se establece según Mondolfo a partir no del encadenamiento de los sistemas, sino de la propuesta, desarrollo y vicisitudes peculiares de los 
problemas filosóficos. Lo que verdaderamente permanece a lo largo de la historia de la filosofía son sus problemas.

La concepción de Mondolfo se vincula con la hegeliana en diversos puntos. En primer término reconoce con Hegel la necesidad de postular la continuidad en el desarrollo histórico de la filosofía cuando dice que hay en esa concepción una "valorización de la historia muy eficaz, que se afirma de una manera muy enérgica contra todo antihistoricismo, toda concepción negadora de la continuidad histórica, toda aseveración de la arbitrariedad y accidentalidad del proceso histórico". ${ }^{5}$

Si bien el origen de la tesis de Mondolfo se halla en el propio Hegel y representa, como ya veremos, otro enfoque historicista, sin embargo se aparta de él aunque no sin reconocer su singular aportación a la historia de la filosofía en cuanto logró mostrar que "en el proceso de la historia de la filosofía los sistemas no surgen al azar, sin razón o fundamento histórico. Su sucesión lejos de presentarse inconexa está íntimamente conexa en su desarrollo". ${ }^{6}$

La separación está fundada en la consideración de que si bien efectivamente, como decía Hegel, hay una conexión necesaria entre cada uno de los momentos del desarrollo histórico, por lo que hace a la historia de la filosofía, Mondolfo señala que: "la vinculación que existe entre cada momento del desarrollo histórico y los anteriores y sucesivos consiste en la profundización y desarrollo progresivo de los problemas planteados por la conciencia filosófica".?

Si bien visto se debe reconocer una necesidad en la vinculación de esta cadena problemática, por otro lado para Mondolfo es innegable la existencia de "una contingencia que permite irregularidades e interrupciones, no menos que una multiplicidad de desarrollos paralelos y procede de la libre espontaneidad del espíritu de cada filósofo". ${ }^{8}$

Dentro de su perspectiva de la historia de la filosofía, Mondolfo nos previene contra dos errores:

1. Considerar a la historia de la filosofía como sucesión incoherente de sistemas que se contradicen y destruyen. Es decir, asumir la propuesta de una historia sin continuidad que desconocería, por una parte, el vínculo entre los momentos sucesivos del desarrollo histórico de la filosofía y, por otra, no percibiría la continuidad consistente justamente en la profundización progresiva de los problemas filosóficos.

2. El segundo error consistiría en aceptar la idea de que el desarrollo histórico está dominado por una necesidad interior absoluta (al modo

5 Ibid., pp. 53-54.

6 Ibid., p. 54.

7 Loc. cit.

8 Loc. cit. 
de Hegel) que convierte a la historia de la filosofía en preparación necesaria de un sistema final.

Así pues, Mondolfo propone la confluencia de necesidad y contingencia en el proceso histórico de la filosofía. La necesidad estaría representada por los antecedentes indispensables para determinar un desarrollo ulterior en la filosofía; sin embargo, tales condiciones necesarias no son suficientes a pesar de su fecundidad potencial y se hacen realidad solamente cuando encuentran en algún pensador el terreno adecuado.9

Naturalmente que en esta concepción salta a la vista el problema de la relación necesidad-contingencia, así como el que la contingencia esté representada únicamente por una "libertad espiritual" del individuo y no se especifique el papel que jugarían otras condiciones externas, culturales, sociales, económicas, políticas, etc.

El esquema resultante es al parecer el encadenamiento de problemas condicionado por una necesidad interna en que los antecedentes son los condicionantes necesarios, con lo cual se subraya la continuidad de la filosofía en el sentido de una lógica interna, pero se abre la posibilidad de una explicación desde fuera al estipular la insuficiencia de las condiciones, por lo que esta cadena viene a ser impactada, fragmentada o accidentada por la libertad espiritual del individuo, el filósofo que profundiza en los problemas.

La idea de que debe otorgarse prioridad al desarrollo de los problemas de la filosofia y no a la sucesión de los sistemas, si se sostiene la continuidad en la historia de la filosofía, se fundamenta para Mondolfo en que el desarrollo progresivo de los problemas exhibe siempre un carácter de continuidad que no ofrece la totalidad del sistema y, por otra parte, la destrucción del sistema implica casi siempre la profundización de la conciencia de los problemas.

Desde una perspectiva más general, Mondolfo encuentra que las relaciones entre la filosofía y otros campos de la cultura humana son consecuencia de la unidad del espíritu y de sus actividades y conocimientos, razón por la cual tampoco hay barreras para la comprensión de los problemas filosóficos entre las épocas más distantes puesto que existe una unidad y continuidad del desarrollo del espíritu. ${ }^{10}$

Mondolfo hace suya la afirmación de Croce de que "el espíritu humano es historia... así el espíritu encierra en sí toda su historia y coincide luego consigo mismo"."11

Con base en lo dicho, Mondolfo afirma que la historia "ofrece al proceso de nuestra formación espiritual y llega hasta nuestra época y

9 Cfr. ibid., p. 55.

10 Cfr. ibid., p. 93.

11 Ibid., p. 93. 
actúa en nuestro interior... y vive todavía en nosotros". ${ }^{12}$ Gracias a semejante concepción se justifica la búsqueda histórica como reconstrucción, la cual implica un buen grado de subjetividad, situación acorde con esta concepción si se recuerda que nuestro espíritu es una formación histórica y la historia no es más que el desarrollo del espíritu.

\section{La continuidad histórica de la filosofía}

La expresión de la continuidad histórica de la filosofía en la alternancia de periodos problemáticos y periodos sistemáticos nos ha parecido fundamental en relación con el problema de la continuidad histórica de la filosofía. Paso a exponer mi punto de vista.

En principio recojo la idea de que existe una continuidad en la historia de la filosofía a la par que asumo que ésta no puede ser entendida sino como parte de la historia total del hombre. Por otro lado, no participo ni del subjetivismo de la reconstrucción histórica ni del objetivismo que anula la búsqueda de una explicación y aun más de una interpretación histórica. Dicho lo cual trataré de exponer cómo entiendo la continuidad en la historia de la filosofia. Para efectos del análisis expondré primero la mecánica o lógica de la continuidad en la actividad filosófica para más tarde explicar cómo se enlaza con las condiciones externas.

Encuentro que existe en efecto un desarrollo continuo de la actividad filosófica que puedo caracterizar de momento muy formalmente como una actividad metódica y sistemática, actividad que parece discurrir por dos vías de realización básica, a saber: la elaboración sistemática y la elaboración problemática, fases que en la actividad misma no se excluyen, ya que sistematicidad y problematicidad son notas constitutivas de este hacer. No obstante, para el análisis que aquí nos ocupa es fundamental la distinción entre ambas características, ya que me propongo distinguir en la continuidad histórica de la filosofía entre periodos eminentemente sistemáticos y periodos eminentemente problemáticos.

En el ámbito propio de la actividad filosófica, la actitud sistematizadora pretendería la unificación sobre principios fundantes y organizativos de las formaciones teóricas más significativas que le fueran contemporáneas; al paso que la actitud problematizadora buscaria revisar, poner en crisis, desechar o sustituir tales principios a fin de proporcionar una base mejor a las nuevas formaciones teóricas.

Semejante alternancia de periodos preferentemente problemáticos con periodos preferentemente sistemáticos no halla su cabal explicación en 
el mero proceso interno del despliegue filosófico ni tampoco puede entenderse como meramente casual, sino que estas manifestaciones de la actividad filosófica concuerdan significativamente en los periodos de consolidación o crisis de las formas económico-sociales adoptadas por las distintas comunidades humanas. La relación naturalmente no es inmediata ni explicable sólo por la determinación de la forma económicosocial, pero parece haber una relación más o menos obvia entre una hegemonía político-cultural o la consolidación de una forma económico-social en un Estado y el florecimiento, en términos generales, de los sistemas. Por el contrario, es manifiesto que en los periodos de crisis la actividad filosófica se desarrolla preferentemente como problematizadora en dos aspectos, ya denunciando la insuficiencia de los sistemas explicativos a la luz de nuevos descubrimientos, ya introduciendo nuevos fundamentos o principios a la luz de nuevos enfoques o cambios de perspectiva que se plantean en el interior mismo de los viejos sistemas.

El esquema aquí esbozado guarda cierta semejanza con la idea expresada por el maestro Ramón Xirau de que en la historia de la filosofía pueden detectarse periodos de "acarreo" y periodos de "grandes sumas o sintesis". De este planteamiento parece desprenderse que el sistema es en forma retrospectiva la base de la explicación de todo un periodo que le precede y que parece entenderse como etapa acumulativa de elementos que vendrian a integrarse en la síntesis. Mi punto de vista difiere de tal concepción en la medida en que me parece que ambas formas del despliegue de la actividad filosófica, que se manifiestan alternativamente en los periodos problematizadores o sistematizadores, tienen como producciones la misma importancia, de modo que yo reconocería una mutua condicionalidad entre ellas, por lo que el peso de la explicación no recae exclusivamente sobre una de las dos manifestaciones de la actividad filosófica.

Por otra parte, el término "acarreo" sugiere únicamente el aspecto aportativo de la fase previa a la síntesis pero no enfatiza suficientemente el problema de la superación de viejas o anquilosadas teorías que sucumben total o parcialmente al golpe de la crítica.

Por último deseo aclarar, en cuanto al esquema propuesto, que se hace necesario distinguir entre lo que podríamos denominar estrictamente como las fases de la actividad filosófica, y que se refieren al carácter metódico y sistemático de cualquier creación filosófica, y las etapas o periodos alternantes en su historia. Para mayor claridad de este aspecto proponemos la distinción que establece Cassirer entre "espiritu de sistema" y "espíritu sistemático" para caracterizar respectivamente a la Filosofía Moderna y a la Filosofía de la Ilustración. El primero hace alusión a que de manera general la Filosofía Moderna tiende a la crea- 
ción de sistemas totalizadores que buscan, a la par que fundamentar, integrar todo el ámbito del conocimiento humano, al paso que el siglo xvirI rompe con el esquema totalizante y busca penetrar sistemáticamente en cada parcela del saber humano fundamentándola, de suerte que se ha denominado a sí mismo el siglo filosófico. Esta consideración de Cassirer nos permite explicar que en lo que entendemos como un periodo preferentemente problematizador no falta nunca la labor sistemática aun cuando no se tenga por meta expresa la elaboración de un sistema, al paso que en las etapas eminentemente sistematizadoras se dan planteamientos problemáticos. Así, no puede hablarse tanto de exclusión cuanto de determinaciones que orientan la labor filosófica más en un sentido que en otro en los diversos periodos.

\section{Intento de caracterización de la historia de la filosofia del Renaci-} miento como periodo eminentemente problematizador

El esquema explicativo acerca de la continuidad en la historia de la filosofía, aunque expuesto en un nivel abstracto y por ende incompleto, antecede a la consideración de la historia de la filosofía del Renacimiento como un periodo de actividad eminente, aunque no exclusivamente, problematizante. Así, la característica más importante del Renacimiento en tanto periodo de actividad filosófica sería su hacer cuestionante, el cual está determinado por ser éste un momento de crisis general como diversos historiadores lo señalan. A. Hauser señala que en el Renacimiento no se da la aparición del hombre nuevo, ni en sentido estricto la nueva concepción del mundo, sino justamente la fluctuación, la crisis del hombre viejo y del mundo tradicional condicionan la aparición del nuevo hombre y la nueva idea de mundo.

Como época de crisis, el Renacimiento ofrece el espléndido espectáculo de una febril actividad intelectual que en casi todos sus desarrollos toca en una $\mathfrak{u}$ otra forma problemas filosóficos sustantes; por ello mismo la organización de semejante caudal ha exigido un esfuerzo extra por parte de los historiadores de la filosofía. Así, Cassirer, al tratar de agrupar los problemas en torno al hombre y al mundo característicos del momento, toma como guía rectora el pensamiento de Cusa en quien encuentra el germen de los problemas filosóficos más importantes del Renacimiento. Pero frente a este intento de sistematización de los problemas hay quien ha recorrido el camino inverso y, profundizando en Ias teorías expuestas por los autores renacentistas, ha rastreado sus fundamentos filosóficos; así es como Jaspers ha encontrado a Leonardo como filósofo. Las vicisitudes metódicas en que la mayor parte de los historiadores de la filosofía se han visto envueltos para proponer una visión articulada del 
Renacimiento, ora sumergiéndose en el arte, la ciencia o la política para detectar sus fundamentos filosóficos, ora mostrando el origen y destino de los problemas filosóficos, esto es, el desarrollo problemático a través de los diversos autores, nos hace afirmar que en efecto lidiamos con un periodo de la filosofía básicamente problematizador. Aun más, la resistencia abierta o disimulada para considerar a autores como Ficino, Pico della Mirandola, Bruno, etc., como verdaderos filósofos, muestra con claridad cuál es la idea que funge como criterio de "auténtica filosofía" (el determinismo sistemático). Así, se alega que Leonardo no se ocupa propiamente de la filosofía, que Pico resulta finalmente tradicionalista y a lo mejor no muy profundo y que Bruno no presenta sino una visión especulativa de la ciencia. A pesar de la aparente diversidad, el argumento de base es uno solo: si no desarrollaron algo semejante a un sistema no son filósofos y, peor aun, si se dedicaron a la teoría pictórica, a la política práctica o a la especulación cosmológica, ¿qué tienen de filósofos?

La segunda objeción secundaria habla de la falta de comprensión histórica. Es no entender ni al hombre ni a su cultura en un momento dado. La primera en cambio es de principio, depende del criterio que se sostenga en relación con el desarrollo y continuidad de la filosofía.

Puede entenderse el hacer problematizador del Renacimiento como una crisis filosófica que se origina en un doble choque:

a) Entre los nuevos descubrimientos y los principios inadecuados para explicarlos.

b) Entre los nuevos enfoques, nuevas pautas teóricas metódicas que desvirtúan la visión general integrada, tradicional del mundo.

En consecuencia, hay un planteamiento problemático en dos sentidos: el descubrimiento de los nuevos hechos, teorías, fenómenos, etc., que no caben en las viejas pautas explicativas y la producción de nuevas pautas no susceptibles de ser aplicadas al mundo en su concepción tradicional. En el primer caso se impone la necesidad de revisar los principios y en el segundo la de revisar los hechos tradicionalmente aceptados. Un ejemplo del primer tipo son Leonardo o Bruno; uno del segundo, Cusa.

Leonardo incursiona en la teoría pictórica que él entiende como verdadera ciencia de la naturaleza, a partir de la cual se puede desplegar didácticamente todo nuestro conocimiento del mundo y plantea la observación y el experimento fundados en la razón como los medios más adecuados para alcanzar el conocimiento de las razones del universo.

Bruno, a partir del examen de las aportaciones de la metafísica al terreno del conocimiento físico y astronómico, las declara absolutamente limitadas para dar cuenta de un nuevo concepto del universo expresado 
para él básicamente en las tesis copernicanas y por ende modifica la concepción aristotélica corriente.

Cusa, por su parte, en el examen de la tradición metafísica y sin ninguna intención de romper con ella, propone nuevos enfoques a los viejos problemas de donde surgen principios nuevos para fundamentar el conocimiento, para la revaloración del mundo natural y la concepción general del hombre, al fundar lo que sus comentaristas llaman la metafísica de la inmanencia.

Tales tensiones y choques de los principios a los hechos o de los hechos a los principios son los que caracterizan la labor problematizadora en el ámbito filosófico. Así, la crisis halla su expresión filosófica en el deslinde y ajuste de hechos y principios explicativos en que se origina una amplia gama de problemas. Los desarrollos de tales problemas en el Renacimiento son más o menos metódicos, pero si se antojan incompletos es porque expresan el cambio del viejo método -que se cuestiona por sustentarse en el argumento de autoridad, el consenso universal y la silogistica aristotélico-tomista, como auténticas formas de adquisición del conocimiento-, a nuevas pautas metódicas que se gestan y comienzan a desarrollarse y van evolucionando paulatinamente y que afectan sustancialmente el carácter del sujeto cognoscente como más autónomo, autoconciente y activo, que culmina en la filosofía cartesiana con quien se inaugura el espíritu de sistema del siglo xvir. 